\title{
A submillimeter exponential disk in M 51: Evidence for an extended cold dust disk
}

\author{
R. Meijerink ${ }^{1}$, R. P. J. Tilanus ${ }^{2}$, C. P. Dullemond ${ }^{3}$, F. P. Israel ${ }^{1}$, and P. P. van der Werf $^{1}$ \\ ${ }^{1}$ Sterrewacht Leiden, PO Box 9513, 2300 RA Leiden, The Netherlands \\ e-mail: meijerin@strw.leidenuniv.nl \\ 2 Joint Astronomy Centre, 660 N. A'ohoku Pl., Hilo, Hawaii, 96720, USA \\ 3 Max-Planck-Institut für Astrophysik, Karl-Schwarzschild-Str. 1, Postfach 13 17, 85741 Garching, Germany
}

Received 18 March 2004 / Accepted 21 September 2004

\begin{abstract}
A $850 \mu \mathrm{m}$ map of the interacting spiral galaxy M 51 shows well-defined spiral arms, closely resembling the structures seen in $\mathrm{CO}$ and $\mathrm{HI}$ emission. However, most of the $850 \mu \mathrm{m}$ emission originates in an underlying exponential disk, a component that has not been observed before in a face-on galaxy at these wavelengths. The scale-length of this disk is $5.45 \mathrm{kpc}$, which is somewhat larger than the scale-length of the stellar disk, but somewhat smaller than that of atomic hydrogen. Its profile cannot be explained solely by a radial disk temperature gradient but requires the underlying dust to have an exponential distribution as well. This reinforces the view that the submm emission from spiral galaxy disks traces total hydrogen column density, i.e. the sum of $\mathrm{H}_{2}$ and $\mathrm{HI}$. A canonical gas-to-dust ratio of $100 \pm 26$ is obtained for $\kappa_{850}=1.2 \mathrm{~g}^{-1} \mathrm{~cm}^{2}$, where $\kappa_{850}$ is the dust opacity at $850 \mu \mathrm{m}$.
\end{abstract}

Key words. Galaxy: general - galaxies: ISM - radio continuum: galaxies

\section{Introduction}

After the introduction of the SCUBA bolometer camera at the JCMT a number of nearby galaxies have been imaged in the submm range to investigate the cold dust component of the ISM (Alton et al. 2002; Israel et al. 1999; Stevens \& Gear 2000). The spatial distribution of dust and its relation to other components of the ISM is best studied in face-on systems, but at submm wavelengths surface brightnesses are typically low. This particularly hampers the search for diffuse emission from inter-arm regions. Moreover, most of these nearby galaxies are larger than the field-of-view of SCUBA, requiring scan-mapping which, as explained below, causes features in the background at the level of a few mJy, frustrating further attempts to determine the level of any weak extended emission. The example of NGC 6946 is illustrative. Little diffuse emission in the inter-arm region was seen by Alton et al. (2002) in the $850 \mu \mathrm{m}$ map. Consequently, they state that "relatively little is known about how the dust is distributed with respect to the spiral arms and, in particular, whether inter-arm grain material is prevalent or no".

At resolutions much lower than those provided by the JCMT, the cold dust in galaxies has been mapped with ISO in the far infrared (Haas et al. 1998; Popescu et al. 2002; Hippelein et al. 2003; Tuffs \& Gabriel 2003; Popescu \& Tuffs 2003). These observations confirm the ubiquitous presence of cold dust below $20 \mathrm{~K}$ first observed by Chini et al. (1986) and indicate that the cold dust component is smoothly distributed over the disk and heated by the diffuse interstellar radiation (Hippelein et al. 2003; Xu et al. 1994). Alton et al. (2001) reported evidence for diffuse inter-arm dust in SCUBA observations of NGC 7331. For a recent discussion of "dusty disks" see also Bianchi (2004).

M 51 is a well studied, face-on interacting spiral galaxy at an assumed distance of $9.7 \mathrm{Mpc}$. It is well known for its strong CO emission, but contrary to NGC 6946 its density-wave and spiral arms are well-organized and prominent, showing a high contrast in the radio continuum (see e.g. Tilanus \& Allen 1991; 1989). The distribution of cold dust in the nuclear region of M 51 was previously mapped at $1.2 \mathrm{~mm}$ by Guélin et al. (1995), who noted a close correlation with CO line emission. Our current $850 \mu \mathrm{m}$ observations (Fig. 1) show that its dust emission is exceptionally strong. The spiral arms are clearly distinguished, but there is also a substantial amount of emission from an extended diffuse disk. To our knowledge, such a submillimeter disk has not been clearly identified in any other galaxy, but in view of the ISO observations discussed previously it may be a common component of spiral galaxies. In this paper we will explore the nature and properties of this disk. The $850 \mu \mathrm{m}$ spiral structure of M 51 will be discussed in a future paper.

\section{Observations and data reduction}

M 51 was observed at $850 \mu \mathrm{m}$ and $450 \mu \mathrm{m}$ in the spring of 1998 and 1999 at the JCMT ${ }^{1}$ using the Submillimeter Common

1 The James Clerk Maxwell Telescope is operated by the Joint Astronomy Centre on behalf of the Particle Physics and Astronomy Research Council of the UK, The Netherlands Organization for Scientific Research and the National Research Council of Canada. 


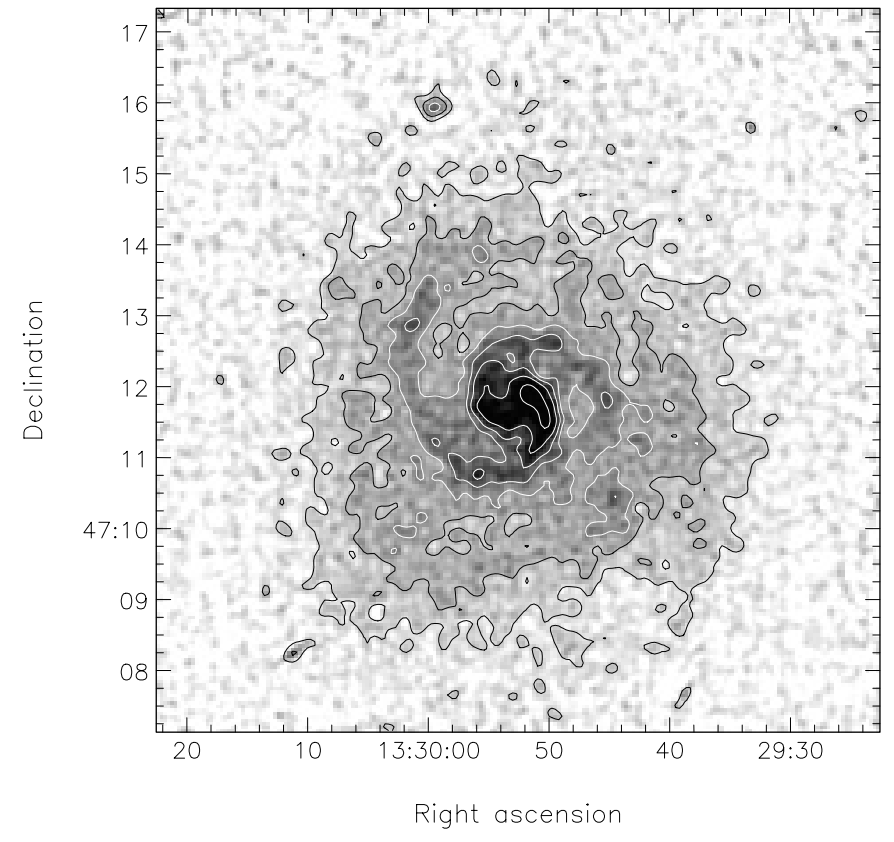

Fig. 1. SCUBA calibrated and background-corrected $850 \mu \mathrm{m}$ greyscale image of M 51 with $850 \mu \mathrm{m}$ contour superimposed. The contour levels are $13.5(=1.6 \sigma), 27,40.5,58.5,72,90$ and $117 \mathrm{mJy} /$ beam. $13.5 \mathrm{mJy} /$ beam corresponds to $2.6 \mathrm{MJy} \mathrm{sr}^{-1}$. This image clearly shows the extended $850 \mu \mathrm{m}$ disk. The small source to the north is the nucleus of NGC 5195.

User Bolometer Array (SCUBA: Holland et al. 1999). In order to map a $13.5^{\prime} \times 13.5^{\prime}$ region around $M$ 51, the camera was used in scan-map mode during which it scanned at a rate of $3^{\prime \prime}$ per second across the field, while the secondary was chopped with a frequency of $7.8 \mathrm{~Hz}$ in right ascension or in declination to cancel atmospheric signal variations as well as bolometer DC fluctuations. This uses the revised version of the Emerson-Klein-Haslam algorithm proposed by Emerson (1995). The scan angle across the field, $\left(15.5 \pm 60^{\circ}\right)$, was chosen such that the resulting image is fully sampled. The total integration time was about $20 \mathrm{~h}$ spread over 6 nights.

To restore the brightness distribution of the source, the chop must be deconvolved from the observed map by using a division in the Fourier plane (Jenness et al. 2000). The Fourier transform (FT) of the chop is a sine wave with zeroes at the origin and at harmonics of the inverse chop throw. The initial observations used chop throws of 20, 30, and 65 arcsec. To minimize regions of low weight (close to zeroes) in the Fourier plane we later added throws of 44 and 68 arcsec. Each individual observation was flat-fielded, corrected for atmospheric opacity, and de-spiked. For each of the 10 different chop configurations the individual maps were co-added. Next, the resulting 10 maps were Fourier-transformed, weighted, and coadded. The coadded image was transformed back to yield the finished image. This is the standard reduction for SCUBA scan-map observations as implemented in the SCUBA User Reduction Facility software (SURF: Jenness \& Lightfoot 1998).

The data were flux-calibrated using Mars and the secondary standards HL Tau and CRL 618. In the final image, the beamsize at $850 \mu \mathrm{m}$ is about $15^{\prime \prime}(F W H M)$. The calibrated image is shown in Fig. 1 and has an overall rms of $\sim 9 \mathrm{mJy} / \mathrm{beam}$. The inconspicuous source to the north is the nucleus of NGC 5195. The familiar spiral pattern is clearly visible and can be traced over a large fraction of the disk. This is quite unlike the submm continuum observations of other face-on spirals, which in general lack the sensitivity to trace the arms outside the inner region. In detail, the morphology of the arms resembles closely the one seen in CO emission (Aalto et al. 1999; Wielebinski et al. 1999), and we will address the contamination of the continuum emission by in-band line emission in Sect. 3 .

Remarkably, the $850 \mu \mathrm{m}$ emission from M 51, showing the distribution of the cool dust, is dominated by a diffuse exponential disk, a component that has not been directly imaged before at submm wavelengths and with a high resolution in a face-on spiral. The $450 \mu \mathrm{m}$ observations also show spiral structure and an extended disk. In part because of the difficulty of deriving an accurate flux calibration and the poorer image quality we will not discuss further the $450 \mu \mathrm{m}$ data in this paper.

\subsection{Sky noise and background removal}

Analyzing extended structures in SCUBA scan maps is a delicate process. While there is no doubt about the reality of the exponential disk, careful processing is required for reliable parameter extraction. There are a number of reasons for this.

Firstly, while regions of low weight have been minimized through the use of many different chop configurations, the zero near the origin of the Fourier plane cannot be suppressed. This results in a poor determination of the largest-scale features in the map, including the total flux density. The effect is similar to that caused by missing short spacings in radio interferometry observations and it has a similar effect on the background.

Secondly, chopping removes noise due to sky fluctuations with a frequency comparable to the chop frequency. It cannot remove the effect of a sky signal which is steadily increasing or decreasing during the scan, i.e. a slowly and monotonically varying component of the sky. In such situations the "on" minus "off" will always leave a small residual sky signal. We have used the standard SURF option, scan_rlb, to subtract a linear baseline from each bolometer scan fitted to the outer 2 arcmin on each side of the scan. While this significantly improves the flatness of the background, it will leave any ripples that may be due to non-linear variations of the sky during each scan. To further improve the sky-noise removal we have used the SURF routine calcsky (Jenness \& Lightfoot 1998), which is similar to the remsky routine used for non-raster data. A detailed discussion of these methods is beyond the scope of the paper, but while $s c a n \_r l b$ relies on the fact that there is no source signal at the edges of the map, calcsky makes use of the fact that (a component of) the sky noise is correlated across the bolometer array during each integration step. The application of both methods improved the flatness of the background without changing the overall appearance of the image.

Nevertheless, even with the application of the above, relatively objective, methods, background artifacts remained, likely because of the missing Fourier zero. This is a common feature of SCUBA scan maps. We used an iterative procedure 
Table 1. Galaxy parameters.

\begin{tabular}{|c|c|c|}
\hline & M 51 & \\
\hline Type $^{a}$ & SAS(s)bcp & \\
\hline \multicolumn{3}{|l|}{ Radio centre: } \\
\hline $\mathrm{RA}(\mathrm{B} 1950)^{b}$ & $13^{\mathrm{h}} 27^{\mathrm{m}} 46.3^{\mathrm{s}}$ & \\
\hline $\operatorname{Dec}(\mathrm{B} 1950)^{b}$ & $+47^{\circ} 27^{\prime} 10^{\prime \prime}$ & \\
\hline $\mathrm{RA}(\mathrm{J} 2000)^{b}$ & $13^{\mathrm{h}} 29^{\mathrm{m}} 52.7^{\mathrm{s}}$ & \\
\hline $\operatorname{Dec}(\mathrm{J} 2000)^{b}$ & $+47^{\circ} 11^{\prime} 42^{\prime \prime}$ & \\
\hline$V_{\mathrm{LSR}}^{c}$ & $+464 \mathrm{~km} \mathrm{~s}^{-1}$ & \\
\hline Inclination $i^{c}$ & $20^{\circ}$ & \\
\hline Position angle $P^{c}$ & $170^{\circ}$ & \\
\hline Distance $D^{d}$ & $9.7 \mathrm{Mpc}$ & \\
\hline Scale & $21^{\prime \prime} / \mathrm{kpc}$ & \\
\hline Scale-lengths (disk): & $(\operatorname{arcsec})$ & $(\mathrm{kpc})$ \\
\hline$B^{e}$ & $92.8 \pm 9.4$ & $4.36 \pm 0.45$ \\
\hline$I^{e}$ & $81.2 \pm 7.0$ & $3.81 \pm 0.33$ \\
\hline$R^{e}$ & $80.4 \pm 6.1$ & $3.77 \pm 0.29$ \\
\hline$K^{e}$ & $87.1 \pm 6.8$ & $4.09 \pm 0.32$ \\
\hline Effective radius (bulge): & $(\mathrm{kpc})$ & \\
\hline$B^{f}$ & $0.94 \pm 0.72$ & \\
\hline$V^{f}$ & $0.98 \pm 0.52$ & \\
\hline$R^{f}$ & $1.07 \pm 0.57$ & \\
\hline$I^{f}$ & $1.06 \pm 0.50$ & \\
\hline
\end{tabular}

Notes:

${ }^{a}$ RSA (Sandage \& Tammann 1987).

${ }^{b}$ Turner \& Ho (1994).

${ }^{c}$ Tully (1974).

${ }^{d}$ Sandage \& Tammann (1975).

${ }^{e}$ Beckman et al. (1996).

${ }^{f}$ Laurikainen \& Salo (2001).

to correct the image which involved subtracting an inclined exponential (the diffuse disk), un-sharp masking and blanking of the remaining source signal (arms, nucleus), followed by a polynomial plane fit and convolution to obtain a smooth, largescale representation of the background. The operations were done using the finished coadded image. While comparable to the procedure used by e.g. Alton et al. (2002), it is subjective in nature. As guidelines we used the following assumptions: the background is flat and the inter-arm emission originates from the diffuse disk only.

The exponential diffuse disk will be discussed separately in Sect. 4: we fitted a scale-length of $1.95^{\prime}$, perhaps fortuitously the same as found for the $20-\mathrm{cm}$ "base" disk (Tilanus et al. 1988), the inclination and position angle as in Table 1, and fitted a peak of $66 \mathrm{mJy} /$ beam. Across the disk of the galaxy the fitted background approximates a linear slope of $-12 \pm 10 \mathrm{mJy} /$ beam: from -2 in the south to -22 in the north. The mean level of $-12 \mathrm{mJy} /$ beam corresponds to (minus) $9 \%$ of the $132 \mathrm{mJy} /$ beam peak in the raw map. By inspecting the resulting inter-arm regions (Fig. 2) we estimate the local background to be accurate to a level of about $\pm 2 \mathrm{mJy} /$ beam or about $\pm 5 \%$ of the typical knots in the arms. Given the overall $\mathrm{rms}$ in
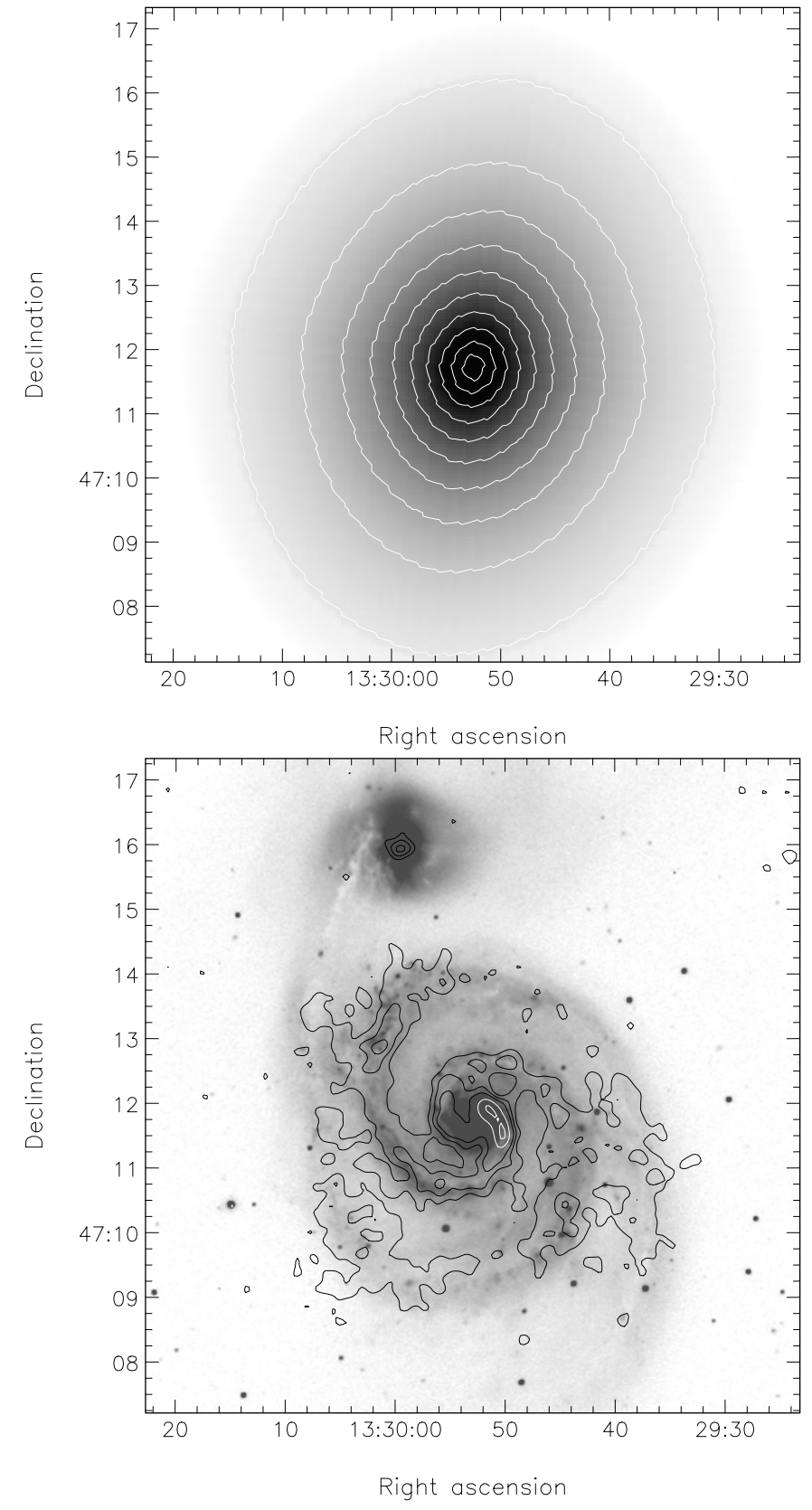

Fig. 2. Top: fitted exponential disk subtracted from the total $850 \mu \mathrm{m}$ emission as shown in Fig. 1. Contour steps are $6 \mathrm{mJy} / \mathrm{beam}$ $\left(1.15 \mathrm{MJy} \mathrm{sr}^{-1}\right)$. The maximum intensity of the disk is $66 \mathrm{mJy} / \mathrm{beam}$ with a major axis scale-length of $1.95^{\prime}$. Bottom: contours of $850 \mu \mathrm{m}$ in M 51 after subtraction of the exponential disk superimposed on a $6400 \AA ̊$ optical image (Tilanus et al. 1988). Contour levels are 12 $\left(=1.5 \sigma ; 2.3 \mathrm{MJy} \mathrm{sr}^{-1}\right), 24,36,56$ and $72 \mathrm{mJy} /$ beam. The image shows the centroid of the $850 \mu \mathrm{m}$ emission to be located along the inner edges of the spiral arms and the optical dust lanes.

the image of $9 \mathrm{mJy} /$ beam, this uncertainty is about four times larger than the statistical uncertainty of the mean over the areas inspected. Figure 1 shows the calibrated observations after correction for the low-level large-scale background fluctuations. 


\subsection{Total flux density}

Using an aperture of $8^{\prime}$, which just excludes the nucleus of NGC 5195, we find an integrated flux of $14.5 \pm 1.1 \mathrm{Jy}$. The uncertainty quoted indicates the range of values found when varying the aperture from $7.4^{\prime}$ to $10^{\prime}$, not the uncertainty resulting from the background variations. To estimate the latter we inspected the variation of the mean level in $2.5^{\prime}$ square regions around M 51, similar to the size of the nucleus and inner arms. This variation is about $0.5 \mathrm{mJy} /$ beam and can serve as an indication of the quality of the background correction where it is applied to regions already largely free from emission. By contrast we note that the $\pm 2 \mathrm{mJy} /$ beam uncertainty of the background in the inter-arm regions, as mentioned in the previous section, is positive in the south and negative in the north, suggesting that the uncertainty of the zero level taken over the whole of the disk of M 51 is less than $2 \mathrm{mJy} /$ beam. Based on these considerations we adopt an overall uncertainty in the background level of $1.5 \mathrm{mJy} / \mathrm{beam}$, which corresponds to $1 \mathrm{Jy}$ when summed over the $8^{\prime}$ aperture. Finally we need to add a $10 \%$ uncertainty in the flux of the calibrators at submm wavelengths. Treating the various uncertainties as statistical errors, we derive a total $850 \mu \mathrm{m}$ flux for M 51 of $15 \pm 2 \mathrm{Jy}$. This value includes the contribution from line emission within the passband of SCUBA's $850 \mu \mathrm{m}$ filter, a topic which will be discussed in the next section.

\section{Contamination by $\boldsymbol{J}=\mathbf{3}-2 \mathrm{CO}$ line emission}

The broad passband of the SCUBA instrument includes the wavelength of the $J=3-2{ }^{12} \mathrm{CO}$ line. Thus, at any point in the map the continuum emission measured by SCUBA is, in principle, contaminated by $\mathrm{CO}$ line emission. In order to investigate the extent of the contamination we obtained with the JCMT in spring 2001 a set of $\mathrm{CO}(3-2)$ spectra across both inner arms of M 51 and supplemented these with archived spectra of the nuclear region. In the arms, the $\mathrm{CO}$ line contribution can be up to 30-40 percent of the observed continuum emission after subtraction of the exponential disk. Thus, a substantial fraction of the arm morphology seen in the SCUBA map directly results from contaminating line emission. Because of the apparently close correspondence between $\mathrm{CO}$ and dust emission (Guélin et al. 1995), the effect is mostly quantitative and does not change the overall morphology of the arms qualitatively. The $J=3-2$ emission from the inter-arm regions is weak and in individual pointings does not exceed the noise in the measurements. In the center of M 51 we find an upper limit of 12 per cent $(3 \sigma)$ to any line contribution to the emission from the fitted disk. Consequently, we may assume either a constant $\mathrm{CO}$ contribution at any level below 12 per cent, or a maximum contribution of 12 per cent at the center decreasing to zero at the outer edge. These two possibilities thus define lower and upper limits, respectively, to the $850 \mu \mathrm{m}$ emission scale-length.

\section{The diffuse disk}

We have separated emission in the diffuse disk from arm emission by subtracting an inclined exponential disk and making the assumption that the inter-arm emission originates from the diffuse disk only (see also Sect. 2.1). The best fit to the data is given by an exponential disk with a maximum intensity of $66 \mathrm{mJy} /$ beam, and a major axis intensity scale-length of $1.95^{\prime}$, corresponding to a linear scale-length of $5.45 \mathrm{kpc}$ at the assumed distance of M 51. As $1 \mathrm{MJy} \mathrm{sr}^{-1}=5.22 \mathrm{mJy} / \mathrm{beam}$, the maximum intensity $66 \mathrm{mJy} / \mathrm{beam}=12.7 \mathrm{MJy} \mathrm{sr}^{-1}$. The subtracted exponential disk and the arm emission are shown in Fig. 2. The global parameters adopted for M 51, such as the distance, inclination, and position angle, are given in Table 1.

The apparent existence of a diffuse exponential disk raises further interesting questions. What kind of dust distribution can account for such a disk: an exponential dust distribution at a constant temperature, a constant dust distribution with a temperature gradient, or both an exponential distribution and a temperature gradient? What is the dust mass in proportion to the amount of gas?

\subsection{The Monte Carlo radiation transfer code RADMC}

To answer these questions, we have modeled the observed emission using a modified version of the axi-symmetric Monte Carlo code RADMC (see Dullemond \& Dominik 2004). This continuum radiative transfer code is based on the method of Bjorkman \& Wood (2001) and Lucy (1999), but adapted to suit the purpose of this paper. The basic idea of this method is as follows. The input luminosity of the system comes from the population of stars in the galaxy. At every location in the galaxy this is represented by a wavelength-dependent source function with the spectral shape of the local stellar population. Integrated over wavelength and over space this source function yields the total intrinsic bolometric luminosity of the galaxy. Each spatial cell represents a certain fraction of this luminosity, dependent on the spatial distribution of the stars. And within such a cell each wavelength interval represents again a fraction of this luminosity, depending on the local spectral shape of the stellar population. In this way each space-wavelength cell represents a well-defined fraction of the total input energy into the problem.

The total input luminosity is now evenly divided over $N_{\text {phot }}$ photon packages. These packages are launched into the galaxy at random angles from randomly chosen space-wavelength cells, where the probability for each space-wavelength cell to launch this photon is proportional to the fraction of the total input luminosity represented by that cell. Each photon package is launched and performs a random walk through the computational domain until it eventually escapes to infinity. After that, the next photon package is launched.

As these photon packages travel through the computational domain they can experience scattering and absorption events. A scattering event merely changes the direction of the photon package, but an absorption-reemission event can also change its wavelength. The probability function for this random wavelength is $\mathrm{d} P(\lambda) / \mathrm{d} \lambda \sim \partial B_{\lambda}(T) / \partial T$, where $T$ is the local dust temperature at the moment the photon package enters the cell. The dust temperature changes during the simulation, increasing with the number of photon packages that have entered the 
cell. Each photon package travelling through a cell increases the "energy" of the cell proportionally to the energy of the photon package, the length $l$ of the path through the cell and the opacity of the cell at that wavelength. The temperature of the cell is increased after each passage in such a way that the total dust emission of the cell $4 \pi \int_{0}^{\infty} \rho_{\text {dust }} \kappa_{\lambda} B_{\lambda}(T) \mathrm{d} \lambda \times V_{\text {cell }}$ (where $V_{\text {cell }}$ is the cell volume) equals the sum of all contributions $\rho_{\text {dust }} \kappa_{\lambda} l L_{\text {tot }} / N_{\text {phot }}$ of all photons that have so far passed through the cell.

The increase of the energy of the cell happens each time a photon travels through the cell, while a discrete scattering or absorption-reemission event (which changes the direction and/or wavelength of a photon package) occurs only at random locations along the path of the photon package. This random location is chosen to be at an optical depth of $\tau=\left|\log \left(\operatorname{ran}\left(i_{\text {seed }}\right)\right)\right|$ away from the last discrete event, where $\operatorname{ran}\left(i_{\text {seed }}\right)$ is a random number between 0 and 1 .

Once all the photons have been launched and have left the system, the dust temperature and scattering source function at every location in the galaxy has been determined. Modulo random fluctuations this should be the correct temperature and scattering source term distribution. Now we use a raytracing code (part of the radiative transfer package RADICAL (Dullemond \& Turolla 2000)) to produce the desired images and/or spectra. But to verify whether this temperature and scattering source term distribution is indeed the correct solution, RADMC has been tested in two ways. First, we have verified that flux is conserved to a high level of precision $(<1 \%)$ by making spectra at many inclination angles and integrating over wavelength and inclination to obtain the total output luminosity. Since the spectra are produced using a ray-tracing code, and not by counting the outcoming photon packages (as is the case for the Bjorkman \& Wood code), this energy conservation is not numerically guaranteed, and therefore represents a good check on the self-consistency. The code has also been tested against many other codes in a 2-D radiative transfer comparison project (Pascucci et al. 2004).

\subsection{Assumed disk structure}

We assumed that the stellar distribution consists of two components, the exponential disk and the bulge. For the bulge we used a Hubble profile (Reynolds 1913; Hubble 1930), which models the stellar emissivity as:

$$
\begin{aligned}
\mathcal{L}(R, z)= & \mathcal{L}_{\text {disk }} \exp \left(-\frac{R}{h_{\mathrm{s}}}-\frac{|z|}{z_{\mathrm{s}}}\right) \\
& +\mathcal{L}_{\text {bulge }}\left(1+B^{2}\right)^{-3 / 2}
\end{aligned}
$$

in which $B$ is given by the expression:

$$
B=\frac{\left[R^{2}+(z /(b / a))^{2}\right]^{-3 / 2}}{R_{\mathrm{e}}}
$$

where $R$ en $z$ are cylindrical coordinates, $\mathcal{L}_{\text {bulge }}$ and $\mathcal{L}_{\text {disk }}$ bulge and disk emissivities at the center of the galaxy respectively, $h_{\mathrm{s}}$ and $z_{\mathrm{s}}$ the respective stellar scale-lengths and scale-heights, $R_{\mathrm{e}}$ the effective bulge radius and $b / a$ the minor/major axial ratio.

We explored both exponential and constant dust distributions. The parameterization to describe the exponential dust distribution is the same as that used for NGC 891 by Xilouris et al. (1998):

$\rho(R, z)=\rho_{\mathrm{c}} \exp \left(-\frac{R}{h_{\mathrm{d}}}-\frac{|z|}{z_{\mathrm{d}}}\right)$

with $\rho_{\mathrm{c}}$ being the central dust density, $h_{\mathrm{d}}$ the dust distribution scale-length and $z_{\mathrm{d}}$ the dust distribution scale-height. A constant dust distribution was obtained from the same expression by putting the scale-length at infinity.

Extinction coefficients were obtained from the model described by Draine \& Lee (1984). We considered four different silicate-to-graphite ratios in the grains, 0.5:0.5, 0.4:0.6, 0.3:0.7 and $0.2: 0.8$. The particle sizes $a$ were set between 0.005 and $0.25 \mu \mathrm{m}$ with a size distribution proportional to $a^{-3.5}$. Our modeling did not include either PAH's or very small dust particles. Although these particles dominate the mid-infrared emission at e.g. 10-20 $\mu \mathrm{m}$, their contribution to submillimeter emission at $850 \mu \mathrm{m}$ is expected to be negligible (see e.g. Li \& Draine 2001).

The spectral energy distribution of the stars was taken from the models calculated by Bruzual \& Charlot (1993). Specifically, we used the model for a continuously star-forming galaxy with a star-forming rate of $10^{-10} M_{\odot} /$ yr per unit solar mass, assuming a galaxy age of $10 \mathrm{Gyr}$. We verified that decreases in the assumed age to 5 Gyr or even 1 Gyr do not cause significant changes in the parameters needed to fit the $850 \mu \mathrm{m}$ radial profile. The dust temperature rises marginally, causing a small change in the dust surface density. The latter, however, is much more influenced by the uncertainty in the total luminosity.

\section{Results and analysis}

\subsection{51 stellar parameters}

First, the scale-length of the stellar emission must be determined as accurately as possible, because it dominates determinations of the scale-length of the $850 \mu \mathrm{m}$ dust emission. The stellar emissivity of M 51 has been extensively studied by Beckman et al. (1996) who determined both arm and inter-arm scale-lengths in the $B, R, I$ and $K$ photometric bands, where they did not correct for extinction. Their inter-arm results are summarized in Table 1. Emission in the $R$ and $I$ bands predominantly traces old stars which are distributed more or less homogeneously through the disk. We used these bands to determine the scale-length of the stellar emission. As this emission is susceptible to dust attenuation, we have taken extinction into account as well. Starting with an initial estimate of the intrinsic scale-length of the stars, the radiation transfer code computes a value for the attenuated scale-length of the stars, which was then compared with the observations.

The M 51 bulge effective radius was not determined. However, as it does not dominate the radial profile, we used a value of $1.03 \mathrm{kpc}$ characteristic of similar galaxies (Laurikainen \& Salo 2001), also summarized in Table 1.

Unlike the scale-length of the exponential star distribution, the scale-heights of both the stellar disk and the dust are in effect degenerate: model and data do not provide useful 
Table 2. Model results.

\begin{tabular}{llll}
\hline \hline CO contamination & No & Maximal & Variable \\
\hline$h_{\mathrm{s}}(\mathrm{kpc})$ & 3.15 & 3.15 & 3.15 \\
$h_{\mathrm{d}}(\mathrm{kpc})$ & 7.65 & 7.65 & 8.65 \\
$\tau_{V, f}\left(L=0.8 \times 10^{11} L_{\odot}\right)$ & 4.8 & 4.1 & 4.2 \\
$\tau_{V, f}\left(L=1.1 \times 10^{11} L_{\odot}\right)$ & 4.4 & 3.7 & 3.7 \\
$\tau_{V, f}\left(L=1.4 \times 10^{11} L_{\odot}\right)$ & 4.0 & 3.4 & 3.5 \\
\hline
\end{tabular}

constraints. For instance, a wide range of stellar scale-heights yields almost identical $850 \mu \mathrm{m}$ emission profiles. Dust temperatures are marginally affected, requiring some changes in the $10-100 \mu \mathrm{m}$ wavelength range of the spectrum. We kept the ratio $h_{\mathrm{s}} / z_{\mathrm{s}}$ identical to that found in NGC 891 (Xilouris et al. 1998). Obviously, as long as the face-on optical depth is kept constant the submillimeter emission does not change perceptibly either when we vary the dust scale-height. When we increase dust scale-heights from 0.25 to $0.35 \mathrm{kpc}$, temperatures change by no more than 1-2 degrees.

In order to determine the total luminosity of M 51, we integrated the UV (OAO), visual and far-infrared (IRAS) flux densities as provided in the NASA/IPAC Extragalactic Database, the $170 \mu \mathrm{m}$ point as given by Tuffs \& Gabriel (2003) and our $850 \mu \mathrm{m}$ point. We find a luminosity of $L_{\text {tot }}=1.1 \times 10^{11} L_{\odot}$, which corresponds to a star formation rate of a few $M_{\odot} \mathrm{yr}^{-1}$, with an estimated uncertainty of about $30 \%$. Of this total luminosity, about $5 \%$ originates in the bulge and the remaining $95 \%$ in the exponential disk.

\subsection{Disk parameter determination}

Even if the stellar luminosity and the CO contamination were known, the stellar scale-height, $z_{\mathrm{s}}$, and the dust scale-height, $z_{\mathrm{d}}$, are degenerate. Adopting values as outlined in the previous section, we explored the parameter space over three variables: (i) the total amount of dust, which scales with the central faceon optical depth $\tau_{V, f}$ integrated from infinity to the galaxy center; (ii) the scale-length of the dust distribution $h_{\mathrm{d}}$; and (iii) the scale-length of the stellar disk $h_{\mathrm{s}}$, since the observed scalelength is attenuated by dust. These variables are uniquely determined when the stellar luminosity and the CO contamination are known. Since this is not the case, we identified combinations of parameters that best reproduced the observed $850 \mu \mathrm{m}$ radial profile.

We have summarized the model results in Table 2. An example of a fit is shown in Fig. 3. Note that the model gives a poor fit in the very center. This is caused by the presence of the bulge in the model which provides additional dust heating, hence more submillimeter emission in the center. We only subtracted the disk component from the observed emission.

In Fig. 4 we show the required face-on central optical depth for a range of total galaxy luminosities and CO contributions. For a luminosity $L_{\mathrm{tot}}=1.1 \times 10^{11} L_{\odot}$ these optical depths $\tau_{V, f}$ range from 3.7 (assuming $12 \% \mathrm{CO}$ contamination) to 4.4 (zero contamination). Inclusion of the luminosity uncertainty increases this to a slightly larger range $\tau_{V, f} 3.4-4$.8. The

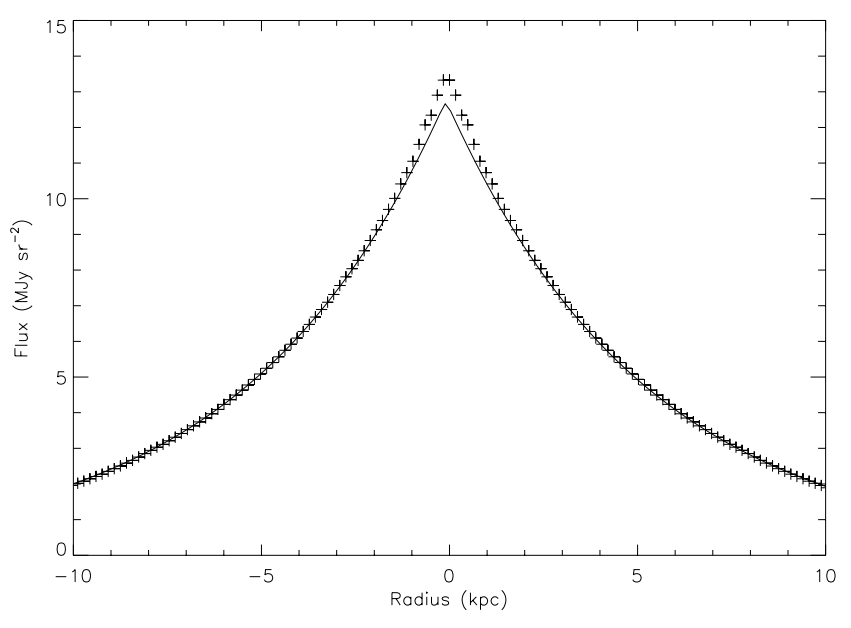

Fig. 3. The model exponential disk (crosses) compared to the actual emission of the M 51 disk (solid line), for $h_{\mathrm{d}}=7.65 \mathrm{kpc}$, $h_{\mathrm{s}}=3.15 \mathrm{kpc}, L=1.1 \times 10^{11} L_{\odot}, \tau_{V, f}=4.35$ and no CO contamination.

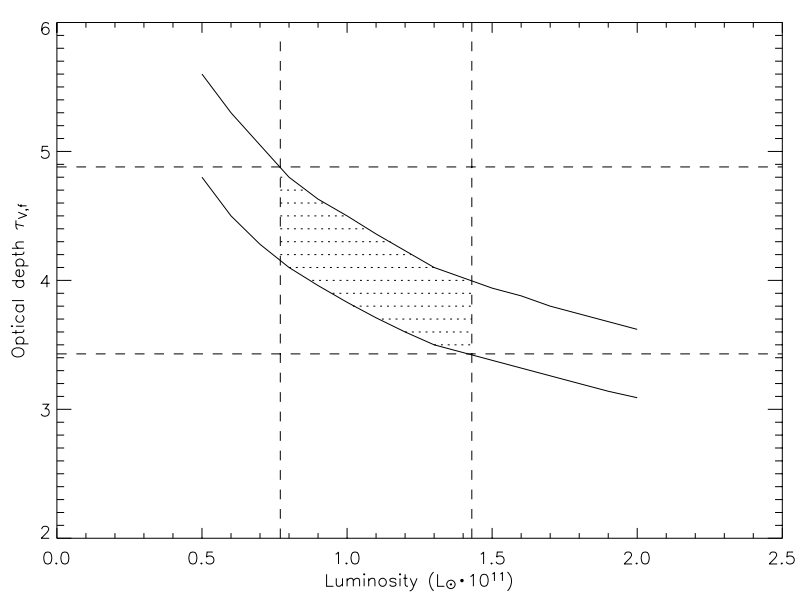

Fig. 4. Resulting optical depth for different luminosities assuming either zero CO contamination (upper line) or an omnipresent maximum 12 per cent contamination (lower line). The shaded area shows the possible solutions.

high optical depth derived here is consistent with the typically higher values derived from far-infrared and submm compared to optical observations (see e.g. Bianchi 2004). Popescu et al. (2000) found a value of 3.1 for NGC 891. In this case, however, arm and interarm emission are fitted simultaneously. The associated scale-lengths vary likewise. For instance, as long as the $\mathrm{CO}$ contamination is constant with radius, we find a dust scalelength $h_{\mathrm{d}}=7.65 \mathrm{kpc}$ and a stellar scale-length $h_{\mathrm{s}}=3.15 \mathrm{kpc}$. On the other hand, if the CO contamination is maximal (12\%) at the center and minimal (zero) at a radius of $10 \mathrm{kpc}$, we obtain a slightly greater scale-length $h_{\mathrm{d}}=8.65 \mathrm{kpc}$. The scale-length $h_{\mathrm{s}}$ remains the same within the uncertainties. The value of $h_{\mathrm{d}}$ is somewhat smaller than the scale-length of atomic hydrogen. The value of $h_{\mathrm{d}}$ is comparable to a scale-length of $9 \pm 1 \mathrm{kpc}$ we derive for the atomic hydrogen outside $6 \mathrm{kpc}$ (Tilanus \& Allen 1991). 


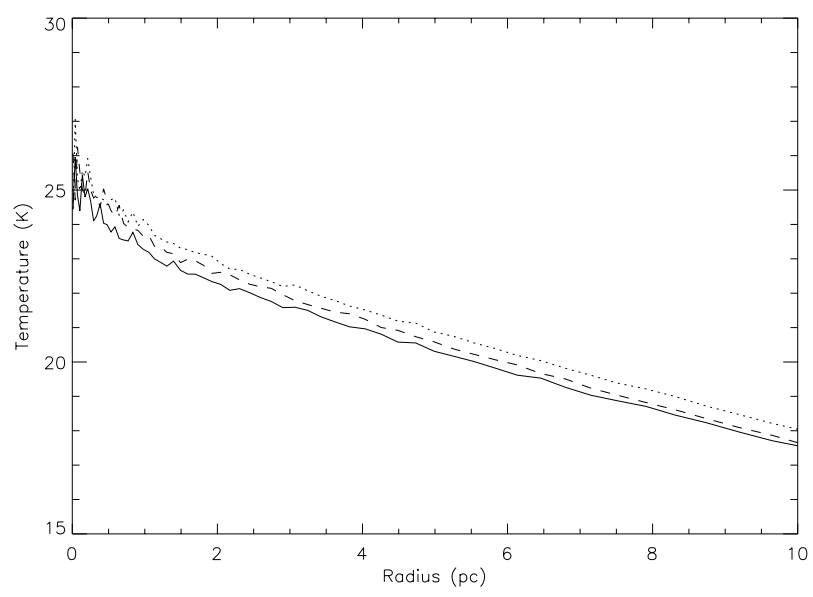

Fig. 5. Radial temperature profile for a total luminosity for M 51 of $L=1.1 \times 10^{11} L_{\odot}$. The solid line marks zero CO contamination, the dotted line a maximum 12 per cent contamination, and the dashed line a contamination decreasing from maximum in the center to zero at the outer edge of M 51.

In addition to a radial density gradient, we also find a radial temperature gradient because the stellar scale-length is much less than the dust scale-length, i.e. the stars are more centrally concentrated than the dust. Radial temperature profiles for the average grain size at position $z=0 \mathrm{kpc}$ are shown in Fig. 5. When the dust scale-length is larger, we also see a steeper temperature gradient through the galaxy. The small fluctuations in the Fig. 5 profile are artifacts due to statistical noise.

The good fit of the $850 \mu \mathrm{m}$ profile provided by exponential distributions with finite scale-lengths already suggests that the M 51 disk is hard to explain in terms of a radially constant density distribution. We nevertheless attempted to force such a fit, and found that this could only be done if the M 51 stellar scale-lengths were more than an order of magnitude less than actually found. Thus, a constant density distribution may be ruled out with confidence. An exponential density distribution of the cold dust is required to explain to observed exponential $850 \mu \mathrm{m}$ disk.

SCUBA submm observations of the edge-on spiral NGC 891 suggest that the cold dust distribution traces the total hydrogen column density i.e. molecular hydrogen in the central regions and neutral hydrogen at larger radii (Fig. 5 in Alton et al. 2000). Subsequent far-infrared observations by Popescu $\&$ Tuffs (2003) have shown that the cold dust emission traced the neutral hydrogen into the extended HI disk. The current observations of M 51, showing an exponential dust density distribution, a close association with the molecular hydrogen in the central region, and comparable scale-lengths of the dust and $\mathrm{HI}$ distributions at larger radii further reinforce the suggestion that the cold dust is correlated with the total hydrogen column density in galaxies.

\subsection{Dust mass and gas-to-dust ratio}

Once the density and temperature profiles are determined we may, in principle, determine the radial dust mass

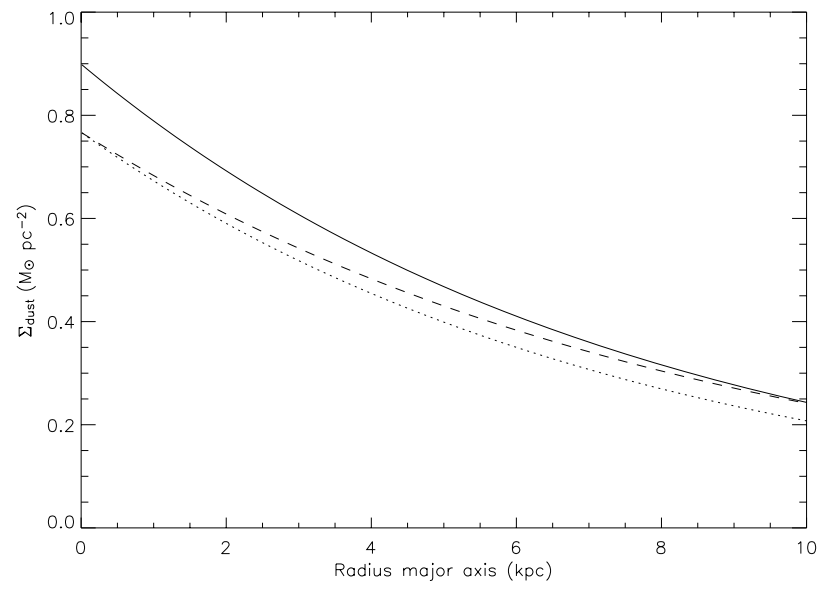

Fig. 6. The dust surface density profiles for a graphite and silicate ratio of 0.5:0.5 and $\kappa_{850}=1.2 \mathrm{~g}^{-1} \mathrm{~cm}^{2}$; no CO contribution (solid line), 12 percent $\mathrm{CO}$ contribution (dotted line) and decreasing $\mathrm{CO}$ contribution (dashed line).

Table 3. Gas to dust-ratios.

\begin{tabular}{lll}
\hline \hline Silicate:Graphite & $\kappa_{850}\left(\mathrm{~g}^{-1} \mathrm{~cm}^{2}\right)$ & Dust:Gas \\
\hline $0.5: 0.5$ & 0.9 & $73 \pm 17$ \\
$0.4: 0.6$ & 1.0 & $82 \pm 21$ \\
$0.3: 0.7$ & 1.1 & $91 \pm 24$ \\
$0.2: 0.8$ & 1.2 & $100 \pm 26$ \\
\hline
\end{tabular}

distribution. In Fig. 6 we show the surface mass densities as a function of radius for a graphite and silicate ratio of 0.5:0.5. The peak dust surface densities are 0.90 (no CO contribution) and $0.77 M_{\odot} \mathrm{pc}^{2}(12$ percent $\mathrm{CO})$. The central gas $\left(\mathrm{HI}+\mathrm{H}_{2}+\mathrm{He}\right.$ ) surface density is $\Sigma_{\text {gas }}=60 \mathrm{M}_{\odot} \mathrm{pc}^{-2}$ (calculated in a future paper), with an estimated uncertainty of 25$30 \%$. This results in a gas-to-dust ratio of $73 \pm 17$.

In this dust mixture, we have a dust emission coefficient of $\kappa_{850}=0.9 \mathrm{~g}^{-1} \mathrm{~cm}^{-2}$. However, the actual value of $\kappa_{850}$ is rather uncertain. James et al. (2002) used SCUBA observations to conclude to a value $\kappa_{850}=0.7 \pm 0.2 \mathrm{~g}^{-1} \mathrm{~cm}^{2}$ but their data show a spread in values for individual galaxies of a factor of two. Worse, Alton et al. (2000) found a three times higher value for the galaxy NGC 891, whereas Agladze et al. (1996) in the laboratory came up with a value about two times higher than that of James et al. (2002).

We can make an estimate of $\kappa_{850}$ in M 51. When we increase the fraction of graphite in the grains, a higher value for the dust emission coefficient is obtained. With a higher dust emission coefficient, the surface mass density will be lower. For $\kappa_{850}=1.2 \mathrm{~g}^{-1} \mathrm{~cm}^{2}$, the peak surface densities as shown in Fig. 6 will be 0.65 (no CO contribution) and 0.56 (12 percent CO). In Table 3, gas-to-dust ratios are shown for four different gas mixtures and for a luminosity of $L=1.1 \times 10^{11} L_{\odot}$. A canonical gas-to-dust ratio of $100 \pm 26$ is obtained with a dust emission coefficient $\kappa_{850}=1.2 \mathrm{~g}^{-1} \mathrm{~cm}^{2}$, somewhat less than twice the value found by James et al. (2002). 


\section{Summary}

1. SCUBA $850 \mu \mathrm{m}$ observations of M 51 show a welldelineated spiral structure superimposed on a prominent extended exponential base disk.

2. The observed base disk is evidence of an underlying exponential density distribution of cold dust in M 51. This reinforces the suggestion that the cold dust in spiral galaxies traces the total hydrogen density, i.e. the sum of $\mathrm{H}_{2}$ and $\mathrm{HI}$.

3. While throughout M 51 a radial temperature gradient occurs, because the stars are more centrally concentrated than the dust, this gradient by itself cannot be the origin of the exponential nature of the observed $850 \mu \mathrm{m}$ disk.

4. A reasonable estimate of the dust emission coefficient, based on a canonical gas to dust ratio of $100 \pm 26$, is $\kappa_{850}=1.2 \mathrm{~g}^{-1} \mathrm{~cm}^{2}$.

5. We find a stellar scale-length $h_{\mathrm{s}}$ of $3.15 \mathrm{kpc}$ and a dust scale-length $h_{\mathrm{d}}$ that ranges from 7.65 to $8.65 \mathrm{kpc}$.

6. We find a typical central face-on optical depth $\tau_{V, f}=4.0$.

Acknowledgements. This research has made use of the NASA/IPAC Extragalactic Database (NED) which is operated by the Jet Propulsion Laboratory, California Institute of Technology, under contract with the National Aeronautics and Space Administration. We want to thank Eric Bell for a helpful discussion about dust opacities. R.T. thanks Ron Allen and Leo Blitz for an invigorating discussion on the nature of the exponential dust disk. We also thank S. Bianchi and the referee, R. J. Tuffs, for their insightful comments and helpful suggestions which have improved this paper.

\section{References}

Aalto, S., Hüttemeister, S., Scoville, N. Z., \& Thaddeus, P. 1999, ApJ, 522,165

Agladze, N. I., Sievers, A. J., Jones, S. A., Burlitch, J. M., \& Beckwith, S. V. W. 1996, ApJ, 462, 1026

Alton, P. B., Bianchi, S., Richer, J., Pierce-Price, D., \& Combes, F. 2002, A\&A, 388, 446

Alton, P. B., Lequeux, J., Bianchi, S., et al. 2001, A\&A, 366, 451

Alton, P. B., Xilouris, E. M., Bianchi, S., Davies, J., \& Kylafis, N. 2000, A\&A, 356, 795

Beckman, J. E., Peletier, R. F., Knapen, J. H., Corradi, R. L. M., \& Gentet, L. J. 1996, ApJ, 467, 175

Bianchi, S. 2004, in Astrophysics of Dust, ASP Conf. Ser., 309

Bjorkman, J. E., \& Wood, K. 2001, ApJ, 554, 615

Bruzual, A. G., \& Charlot, S. 1993, ApJ, 405, 538
Chini, R., Kreysa, E., Kruegel, E., \& Mezger, P. G. 1986, A\&A, 166, L8

Draine, B. T., \& Lee, H. M. 1984, ApJ, 285, 89

Dullemond, C. P., \& Dominik, C. 2004, A\&A, 417, 159

Dullemond, C. P., \& Turolla, R. 2000, A\&A, 360, 1187

Emerson, D. T. 1995, in Multi-Feed Systems for Radio Telescopes, ASP Conf. Ser., 75, 309

Guélin, M., Zylka, R., Mezger, P. G., Haslam, C. G. T., \& Kreysa, E. 1995, A\&A, 298, L29

Haas, M., Lemke, D., Stickel, M., et al. 1998, A\&A, 338, L33

Hippelein, H., Haas, M., Tuffs, R. J., et al. 2003, A\&A, 407, 137

Holland, W. S., Robson, E. I., Gear, W. K., et al. 1999, MNRAS, 303, 659

Hubble, E. P. 1930, ApJ, 71, 231

Israel, F. P., van der Werf, P. P., \& Tilanus, R. P. J. 1999, A\&A, 344, L83

James, A., Dunne, L., Eales, S., \& Edmunds, M. G. 2002, MNRAS, 335,753

Jenness, T., Holland, W. S., Chapin, E., Lightfoot, J. F., \& Duncan, W. D. 2000, in Astronomical Data Analysis Software and Systems IX, ASP Conf. Ser., 216, 559

Jenness, T., \& Lightfoot, J. F. 1998, in Astronomical Data Analysis Software and Systems VII, ASP Conf. Ser., 145, 216

Laurikainen, E., \& Salo, H. 2001, MNRAS, 324, 685

Li, A., \& Draine, B. T. 2001, ApJ, 554, 778

Lucy, L. B. 1999, A\&A, 344, 282

Pascucci, I., Wolf, S., Steinacker, J., et al. 2004, A\&A, 417, 793

Popescu, C. C., Misiriotis, A., Kylafis, N. D., Tuffs, R. J., \& Fischera, J. 2000, A\&A, 362, 138

Popescu, C. C., \& Tuffs, R. J. 2003, A\&A, 410, L21

Popescu, C. C., Tuffs, R. J., Völk, H. J., Pierini, D., \& Madore, B. F. 2002, ApJ, 567, 221

Reynolds, J. H. 1913, MNRAS, 74, 132

Sandage, A., \& Tammann, G. A. 1975, ApJ, 196, 313

Sandage, A., \& Tammann, G. A. 1987, A Revised Shapley-Ames Catalog of Bright Galaxies (Washington: Carnegie Institution of Washington Publication, Carnegie Institution, 2nd ed.)

Stevens, J. A., \& Gear, W. K. 2000, MNRAS, 312, L5

Tilanus, R. P. J., \& Allen, R. J. 1989, ApJ, 339, L57

Tilanus, R. P. J., \& Allen, R. J. 1991, A\&A, 244, 8

Tilanus, R. P. J., Allen, R. J., van der Hulst, J. M., Crane, P. C., \& Kennicutt, R. C. 1988, ApJ, 330, 667

Tuffs, R. J., \& Gabriel, C. 2003, A\&A, 410, 1075

Tully, R. B. 1974, ApJS, 27, 437

Turner, J. L., \& Ho, P. T. P. 1994, ApJ, 421, 122

Wielebinski, R., Dumke, M., \& Nieten, C. 1999, A\&A, 347, 634

Xilouris, E. M., Alton, P. B., Davies, J. I., et al. 1998, A\&A, 331, 894

Xu, C., Lisenfeld, U., \& Voelk, H. J. 1994, A\&A, 285, 19 